\section{Experiences of patients with lung cancer receiving concurrent chemo- radiotherapy}

\section{Eunsook Choi ${ }^{1 *}$ and Sunhee Park²}

\begin{abstract}
${ }^{1}$ National Cancer Center, Educator, Department of Nursing Education, 323 Ilsan-ro, Ilsandong-gu, Goyang-si Gyeonggi-do, 410-769, Republic of Korea
\end{abstract}

${ }^{2}$ National Cancer Center, Head Nurse, Center for Gastric Cancer, 323 Ilsan-ro, Ilsandong-gu, Goyang-si Gyeonggi-do, 410-769, Republic of Korea

\section{Abstract}

Purpose: In an aspect of qualitative treatment, this research gathered lung cancer patients' actual experiences to understand deeply, such as their expectation for treatment results, their difficulties during treatment, and their various requests to their family and medical teams.

Methods: From May to June 2013, semi-structured interviews were conducted with 9 lung cancer patients. Data were collected through a tape-recorded in-depth interview. The analysis of the data was made through the qualitative method.

Results: 124 themes regarding the experience were found. From these 35 concepts, 24 subcategories were emerged. The core category was enduring hardship of the treatment with the hope for full recovery. Six categories included 'Wishing to be cured but concern about recurrence', 'Receiving radiation treatment with pleasure and difficult at the same time'. 'Being sorry for their family's full support and trying to stand alone', 'Having confidence in their medical team', 'regretting for their old days', and 'Wanting to live a long life without illness and pain'.

Conclusion: The results of this study would help oncology nurses to understand the lung cancer patients receiving concurrent chemo-radiotherapy (CCRT) and to develop a quality of life improvement program for physical, psychosocial, and spiritual aspects of nursing.

\section{More Information}

*Address for Correspondence: Eunsook Choi, National Cancer Center, Educator, Department of Nursing Education, 323 Ilsan-ro, Ilsandong-gu, Goyang-si Gyeonggi-do, 410-769, Republic of Korea, 10408, Tel: 031-920-0562; 010-66884887; Fax: 031-920-1908;

Email: eunsookc77@ncc.re.kr

Submitted: February 04, 2021

Approved: March 22, 2021

Published: March 23, 2021

How to cite this article: Choi E, Park S. Experiences of patients with lung cancer receiving concurrent chemo-radiotherapy. Clin J Nurs Care Pract. 2021; 5: 015-023.

DOI: 10.29328/journal.cjncp.1001032

Copyright: (C) 2021 Choi E, et al. This is an open access article distributed under the Creative Commons Attribution License, which permits unrestricted use, distribution, and reproduction in any medium, provided the original work is properly cited.

Keywords: Lung cancer; Concurrent chemoradiotherapy experiences; Qualitative research

D) Check for updates

OPEN ACCESS

\section{Necessity of a research}

In 2012, in Korea, 224,177 cancer cases have occurred, inter alia, lung cancer took over $10.2 \%$ (rank $4^{\text {th }}$ ) of the whole cancer cases as recorded 22,118 cases yearly including both sexes [1].

Unlike the other cancers, lung cancer does not have a specific subjective symptom and a proper early diagnosis, so it is discovered in stage 1 or 2 , and when it comes to the feasible radiation surgery, it is reported less than approximately $20 \%$ among the entire lung cancer patients. Also, because of its high rate of remote metastasis, the cure result is low in spite of trials of personal anticancer treatment based on a surgery, a recently developed radiotherapy, and a molecular targeted cancer therapy. Lung cancer is the number one mortality rate, accounting for $22.9 \%, 18,574$ out of 81,203 cancer deaths. The 5-year survival rate for lung cancer is $32.4 \%$ (2014 2018) which is lower than the total 5-year survival rate, $70.3 \%$ Concurrent chemo-radiotherapy (CCRT) is a treatment to maximize curative effacement obtaining a synergy effect by proceeding chemotherapy and radiotherapy simultaneously [3]. Lung cancer treatment uses complicated and sophisticated radiation dose distribution therapies such as a 3-D conformal radiation therapy (3D-CRT) implementing high-energy X-ray and an intensity modulated radiation therapy (IMRT). It conducts fractional irradiation with 180cGy once a day or 200cGy five times a week using the linear accelerator for about 6 weeks in total, and the total amount of radiation dose would be approximately 6000cGy. On this wise, the local tumor is controlled by radiotherapy, and to prevent relapse of remote organs, chemotherapy is conducted merging with radiotherapy at intervals of 1 or 3 weeks. For CCRT typically the anticancer drug, Cisplatin has been broadly used thanks to its excellent curative effect and radiation sensitizer effect. Due to the toxicity such as esophagitis, however, the research had been required to find 
lower toxicity in drugs, and finally in 1990's the new drugs such as Paclitaxel, Docetaxel, Gemcitabine, Vinorelbine have been used showing high reaction rate and cure rate.

Kang, etc. [4] had analyzed 36 geriatric patients aged over than 65 years old who are treated with CCRT for non-small cell lung cancer(NSCLC) in stage III. And the results showed 11 persons were in IIIA (30.6\%), 25 persons were in IIIB (69.4\%), median age was 70 years old (65-82 years old), median survival rate was 15 months. The 1 year survival rate and 2 year survival rate were was $58.2 \%$, and $31.2 \%$, respectively. Each of relapse-free survival rates was $41.2 \%$ and $19.5 \%$. Besides, radiation pneumonitis above 3 degrees was $13.9 \%$, esophagitis was $11.1 \%$, leukopenia was $5.6 \%$, and anemia was $2.8 \%$.

Noh, etc. [5] had analyzed 633 patients who are treated with CCRT for N2-IIIB NSCLC. The results showed median age was 65 years (range, 36 to 76 years), the median radiation therapy dose was 66Gy in 33 fractions. The most frequent chemotherapy regimen was weekly paclitaxel or docetaxel plus cisplatin or carboplatin (54, 83.1\%). The median follow-up time was 18.8 months. The median and 2-year rates of progression-free survival were 18.8 months and $45.9 \%$, respectively. The median and 2-year rates of overall survival were 28.6 months and $50.1 \%$, respectively. As a more effective treatment to control occult metastasis, Lee, etc. [6] conducted a study on incorporating Erlotinib or Irinotecan Plus Cisplatin into CCRT for Stage III NSCLC according to EGFR mutation status. As a result, patient with sensitive EGFR mutations had significantly longer OS than EGFR-wild patients (74.8 months $v s$. 25.3 months, $p=0.034$ ). In addition, PACIFIC trial, which evaluated durvalumab consolidation therapy after CCRT in inoperable LA NSCLC, demonstrated a statistically significant and clinically meaningful improvement in progression-free survival and a significant improvement in OS. Durvalumab thus provides a new option for patients treated with curative intent [7].

The side effects of radiation therapy are divided into three main phases (early or acute, subacute, and long or chronic effects). Acute effects vary with each patient, are site specific and generally short term, and resolve after completion of treatment [8]. Patients treated with radiotherapy have a fatigue, somnolence, and a sleeping problem. These side effects were reported more seriously when it comes to lung cancer, central nervous system cancer, hematologic malignancy, digestive system cancer, and head and neck cancer rather than prostate cancer and breast cancer [9]. Besides, patients treated with radiotherapy were observed as they have significantly higher tendency toward somatization, depression, anxiety, hostility, etc. compared to the normal control group, and they have lower self-esteem and quality of life [10].

Most of preceding researches for lung cancer patients treated with CCRT focused on cure reaction rate, survival rate, relapse phases, and symptoms [4-7]. The researchers conducted by nursing which study for lung cancer patients with radiotherapy include fatigue, quality of life, and changes of physical symptoms. Research on the effects and side effects of CCRT is essential and very important. It is also very important to understand patients receiving CCRT. In specific, CCRT is a treatment that aims for the full recovery, and thus, there should be an observation and an analysis deeply discussing the patients' expectations of treatment and their adaptation on long-term treatment finding out what kind of difficulties they have. Therefore, this study would help us to develop the understanding of lung cancer patients with CCRT.

\section{Purpose of the research}

The purpose of the research is to deeply understand and figure out the situations with regard to expectations of treatments of lung cancer patients with CCRT, difficulties of treatments, requests for families or medical teams and so on. The research question is "What is getting Concurrent chemoradiotherapy?"

\section{Method of study}

\section{Research plan}

This study is a qualitative research that figures out the actual experience as it is and analyzes the facts to understand comprehensively the treatment experiences of lung cancer patients with CCRT.

\section{Research subjects}

The participants of this study are lung cancer patients with CCRT for 6 weeks respectively selected three by three from the early, the middle and the end of treatment held by proton therapy center of the cancer specialized center located in Gyeonggi-do, thereby 9 in total.

\section{Data collection period and method}

Data collecting was operated through participants' personal interviews as semi-structured interview that the researcher obtained through face-to-face talk using open questions. The interview was held at the counseling office in the proton therapy center, and each time took about 30 to 60 minutes. The data collecting had begun since May $30^{\text {th }}$ 2013 on which authorized by Institutional Review Board (IRB), and it ended June $5^{\text {th }}$ 2013. During the interview the researcher tried not to show the specific attitude such as explanation, judgment or denial, because this subjective opinion can affect participants' statements, but we tried to make empathic atmosphere to make participants to talk their experience freely. Prior to the interview, we collected data with regard to general features of participants and gathered answers by using open questions described below. The interview for data collection proceeded until there is no new content appearing in the contexts but rather become saturated. 
Table 1: Demographic \& Medical Characteristics of Subjects.

\begin{tabular}{|c|c|c|c|c|c|c|}
\hline No. & Histologic type: TNM: Stage & Sex/ Age & No. of Fx/Total No. of Fx & Chemotheray agent & $\begin{array}{l}\text { Dosing } \\
\text { Interval }\end{array}$ & $\begin{array}{l}\text { Duration of diagnosis } \\
\text { (month) }\end{array}$ \\
\hline 1 & NSCLC: SqCC, T2N3M0 stage IIIB & $\mathrm{M} / 57$ & $3 / 25$ & Padexol + Cisplatin & weekly & 3 \\
\hline 2 & NSCLC (ADC: T1bN2M0: stage IIIA) & $\mathrm{F} / 73$ & $22 / 25$ & Etoposide + Cisplatin & q 3 weeks & 2 \\
\hline 3 & NSCLC: SqCC, cT2bN0,stage IIA; inoperable & $\mathrm{M} / 71$ & $4 / 27$ & Paclitaxel + Carboplatin & weekly & 1 \\
\hline 4 & Rec NSCLC (adeno) T1aN2,stage IIIA & $M / 64$ & $21 / 29$ & Padexol + Cisplatin & weekly & 10 \\
\hline 5 & NSCLC(SqCC),T4N2M0, Stage IIIB & $\mathrm{M} / 72$ & $13 / 29$ & Taxol + carboplatin & weekly & 3 \\
\hline 6 & NSCLC(ADC), T4NOM0 stage IIIA & $\mathrm{M} / 72$ & $6 / 25$ & Paclitaxel + Carboplatin & weekly & 1 \\
\hline 7 & Double primary, SCLC (LD) & $M / 68$ & $20 / 29$ & Etoposide + Cisplatin & q 3 weeks & 3 \\
\hline 8 & NSCLC: Adenoca, P/D, TxN2M0, bulky N2 & $\mathrm{M} / 70$ & $3 / 25$ & Padexol + Cisplatin & weekly & 2 \\
\hline 9 & NSCLC (ADC: T2aN2M0) stage IIIA & $M / 65$ & $12 / 29$ & Etoposide + Cisplatin & q 3 weeks & 1 \\
\hline
\end{tabular}

The open questions are as follows.

"What kind of expectations do you have about CCRT?"

"Do you have any questions about the treatment?"

"Do you have any (physical, emotional, spiritual, social, economical difficulties during the treatment?"

"Which part do you think you need a help when you are having a treatment?"

"If you have any requirements from your family, can you tell us what it is?"

"If you have any requirements from the medical team, can you tell us what it is?"

\section{Research analysis}

The recorded data in this study used qualitative content analysis that is used for describing the studied phenomenon frankly and explaining after the dictation. The qualitative content analysis is introspective and interactive as we add new data and edit it continuously through data insight alike the other qualitative research methods. It only allows minimal invasive manner compared to phenomenological method and grounded theory method. This study used traditional content analysis of the qualitative content analysis method, which analyzes and draws a result as a dynamic analyzing form summarizing informational context. In this manner, the study process is conducted as follows.

$1^{\text {st }}$, Transcribe participants' statement as it is from interview recording.

$2^{\text {nd }}$, Obtain intuition and insight about the situation while understanding their experiences as a study participant as familiarized with documents through reading transcribed contents thoroughly.

$3^{\text {rd }}$, Encode extracted meaningful statements. Categorize it to topics by gathering similar extracted codes.

$4^{\text {th }}$, Narrow categorized topics down abstractly and embody its characteristics. At this time, take confirmation from participants and obtain validity of result by taking feedback among researchers to make sure that the research result represents participants' experiences accurately.

\section{Verification of credibility and validity}

We try to retain neutrality by using attentive hearing and parenthesis, because it is possible to sort and judge arbitrarily as researchers are directors of nursing in radiotherapy department and lung cancer ward. Also, we use the method for building credibility represented by Sandelowski [11]. First, we recorded every participant's interview. Second, we transcribed their statements as it is. Third, we selected meaningful statements and encode related contents into the codes and extract topics. Fourth, we took feedback of analyzed results from participants who visited to take therapies. Fifth, we gained verification from a colleague, a chief nurse as an oncology nurse practitioner, and a lung cancer nurse practitioner.

\section{Result}

\section{Characteristics of research participants}

The characteristics of research participants are as described in table 1 . The number of participants was 9, and median age was 70 years old, and activity level was ECOG 1 . Seven participants were receiving outpatient treatment. The other two were taking inpatient treatment, but, one of them was hospitalized due to leukopenia and the other one was taking CCRT with antibiotic treatment because of fever. Two of participants discovered cancer during regular checkups, and seven were started CCRT after diagnosed lung cancer during examination of sudden dyspnea, pleurodynia, weight loss, etc. The number of radiotherapy was below 6 times were four, 12 13 times were two, 20 22 were three by the time of interview. The stages of early, middle and end of treatment were evenly included.

\section{Topics regarding experiences of lung cancer patients with CCRT}

The total number of meaningful statements was 124 that extracted from documents obtained through transcriptions and interviews of 9 participants to observe experiences of lung cancer patients with CCRT. It included each individual's specific situation. And in the next step, we classified similar statements into 18 codes, and condensed sorted codes abstractly embodied characteristics into 6 topics; "Fear of relapse with hoping for full recovery", "Hardship of treatment despite pleasure", "Sense of guilt about sufficient help and 
sense of independence with self-management", "Trust and Following kind medical team", "Regret of past", "Wish for living long and healthy".

\section{Topic 1. Fear of relapse with hoping for full recovery}

The expectation of CCRT was appeared as expectation of full recovery and fear of relapse. Whether they have expectation of full recovery or fear of relapse, participants accepted their current therapy calmly, talked to surrounding people to gain information about treatment and participated related book readings or information sessions.

Hope for full recovery: Seven of participants had hope for full recovery, and they received treatments with an expectation of recovery by success of treatment or confidence in cure by itself. And some of them gained strength from information delivered by surroundings or reading related books as times goes by.

"I aim for nothing else but the recovery. I want full recovery. I think I can get it. I have no idea what doctors have on their mind, but I believe I will recover if I do this and have hope on my mind when I take the treatment." (Participant No.5).

"After two weeks since I heard the news as a shock, I have settled down. Until that, I cried all over, whatever I do, and I had no idea what to do. But, after two weeks with treatment, I could calm down. Listening to others, reading books and stories about cured people....and sitting down...taking treatment...trying to fight over the cancer...but..." (Participant No.9).

Curiosity about treatment progress: They had a curiosity about treatment progress during 6 weeks treatment period. They expressed their longing for hope when they heard positive answers from the medical team.

"I have heard many stories from diverse people when I got here. Compared to whom had took pictures whether the tumor has grown or not, after their three weeks, however, I have not heard any news about how much my tumor has changed. I wonder the reaction of treatment." (Participant No.4).

“For me, I don't know how this will go, and this is suffocating. As a treated person, it is really suffocating. I want some explanations how this is going. It would be great if someone tells me something like 'it gets better slowly' or 'we should wait little bit longer'. But there is no comment about any of that whether it is good or bad. All I can do is following as directed." (Participant No.5).

Fear of relapse: Some of participants had a fear of relapse. They were pleased to take a therapy with a positive thinking, while having a fear of relapse on the other hand. Among them, one participant was trying to gain strength by participating in a lung cancer presentation to get information.
"I heard that lung tends to have a risk of relapse despite of recovery. Sick people talk about it. My friends and all my acquaintances say most of them were died because of relapse. After 4 to 5 years later, it appears again. Many say that if you get over 4 to 5 years, then the risk gets lower. I just hope that there is no relapse for me. (Participant No.2).

"I am curious about the cure, because everybody says it is the most difficult part. So that is why." (Participant No.6).

\section{Topic 2. Hardship of treatment despite pleasure}

The participants with CCRT showed their reactions in 5 codes. The most extinctive reaction among participants was the pleasure of treatment. Besides that, there were side effects of radiotherapy and chemotherapy, difficulties of outpatient treatment, questions about therapy progress and dietary life.

Pleasure of treatment: The participants said that they are pleased to get CCRT with positive thoughts of cure, and feel good about getting continuous treatment. Also, they demonstrated their discomfort derived from CCRT as a natural reaction when the CCRT shows side effects by doing two therapies at the same time.

"I come here with present mind, because I think this is for my cure. It is inevitable. As this disease came to me by chance and is not what I wanted, all I can do is trying to get over it. I think I can get well when I take drugs from doctors. I think I should try, too. That is what I do." (Participant No.1).

"I feel good when I take it. Actually, it feels good. I feel that way by myself. There is no sore spot. It was not hard, because I do the way I am directed. I feel better after the treatment. I eat well. So, I don't have any complaint. Eating well, taking treatment, and no sore spot." (Participant No.3).

"After I am hospitalized, my leukocyte gets back to normal. I recovered just in one day. All were surprised and my hear pounded with excitement... I was happy that my radiotherapy would get in process continuously." (Participant No.9).

Sore throat, fatigue, exhaustion from radiotherapy: They experienced sore throat, sleep disorder, fatigue physically during radiotherapy and expressed their difficulties about visiting center every day.

"Compared to other discomforts, I can endure sore throat, but it is so hard to eat breakfast when I wake up in the morning. It gets better in the afternoon. For example, I have to stretch my back like a stork's neck, if I want to swallow the food at once. I heard the solution is pain killer, but I don't take it, because I can bear this much." (Participant No.7).

“The hardest thing is I can't get sleep at night. I spent the night by laying and sitting. The nighttime is a torture for me. Sometimes, I get out to watch television. I used to have a sleep disorder, but I think it get worse after I got here." (Participant No.3). 
"Everyday...I am tired. Honestly, people may say it is annoying, even if it is for getting a paycheck. I cannot help but go because it is for my disease and cure. It is a really unpleasant thing. It will be 3 weeks if I count this radiotherapy until tomorrow." (Participant No.5).

Nausea, dizziness, lethargy from chemotherapy: The symptoms about chemotherapy are continuous hardness in accordance with anticancer cycle, nausea, lethargy, dizziness, leukopenia.

"My body aches after I get anticancer treatment. The drugs are strong. When I take it, one or two days are okay. For two days I feel okay to eat, but after two days, I feel sick for five to seven days. After this, it gets better as the drug remains low. When it comes to 3 weeks, I gain my appetite back, but it is meaningless due to the fact that I should get the therapy again at this time. It is painful, but other than this, there is nothing else to care." (Participant No.1).

"After first three times, I don't know the exact feeling about it. It was not hurtful. But, taking three times after 3 weeks made me sick enormously as I cannot feel which part hurts the most. I can't say which part hurts, but it is painful. And as time goes by, it gets better after 5 to 6 days. I feel better now and I don't have nausea. By the way, the one who had a therapy for 22 days is suffering now. I will live. I will not die." (Participant No.2)

Difficulties of coming for the therapy everyday: The places that participants go for therapies are various such as a ward, inpatient sanatorium, patient's room, family's house, etc. They manage their difficulties of coming for the therapy every day with a coping plan on their own ways. There was no one who claims he or she would not take the therapy because of this matter.

"I am alone in the sanatorium near MBC broadcasting station. My family is at Jeonju. I have a son who works in Jeonju stayed here for a while. He came here and go, but I said him not to come. I don't know why, but I am doing well guessing my treatment is good. My sanatorium steps helped me to go. But it was uncomfortable, because there was no ward when I get treated." (Participant No.2).

"I'm staying at Uijeongbu (name of city). I come here by taxi. My kids cannot help me to come here due to their jobs. If I should come here once or twice a week, my two sons can drive me here by turns. But I have to come here every day, so they can't help me. Total taxi expenses would be about 250,000 KRW (USD 282) a week. It costs more than my therapy. But my kids don't want me to take a bus. I can't take a bus, also, because I don't have enough strength. I should switch to another bus when I take a bus." (Participant No.5).

“My home is in Busan. Since I have got here, I haven't go my home. I stay in a patient room in front of the center for three weeks. I rent it for a month. It costs 750,000won per month." (Participant No.9).

Curiosity about good and bad food: They were curious about which food is good during the treatment, and they tend to follow orders from medical team. Also, they were curious about nutrition drinks that help them to endure the therapy.

"I wonder which food is bad for the lung cancer. It will be helpful to eat better, if I know which food is good or bad and take notes of it. I don't take food I used to eat. I heard that I should not take meat and sweets a lot. I can't eat sugar? Too many concerns make me have overreactions. I avoid eating meat and sweets, because cancer usually likes to grow if I take them. I received a dog food from Ulsan. It was from my acquaintance that sells dogs, not from the market that raises dogs with dirty treats. It tasted good and had no grease. "(Participant No.4).

Topic 3. Sense of guilt about sufficient help and sense of independence with self-management

Family support is categorized into 3 codes: selfmanagement despite sufficient help from family, sense of guilt to family.

Sufficient help: Participants who have a sufficient help gained strength for their therapy while thinking they must live. During the treatment, they feel love from their families who help them doing their best.

"I get enough help from my families. I am not deserved my family's support considering what I did to my family. They are so good to me. I didn't. They always do. My son asked me to say anything if something is uncomfortable, because it is also helpful for them, too." (Participant No.3).

"I must do my best to live, because my family is so good to me. My kids contribute themselves to this saying it is right to do considering what mom has done to us'." (Participant No.9).

Sense of guilt: Participants have a sense of guilt to their family's full support and their kids who help them every time.

"I need nothing from my family, because I feel bad for them. I am sorry, that's why. Haha. What should I need for my family with this sick body? Haha. I am sorry for them and medical teams. Haha. Because I have a serious disease." (Participant No.1).

Self-management: Participants expressed that they do not want give their family any pressure, because their physical activity rate is ECOG 1, and it means they can do outpatient treatment by themselves and take care of themselves. They feel nervous, however, when family do not help them enough. One of participant had a sense of guilty for his family.

“I don't need anything from my family. I should deal with 
my disease by myself. I don't want give them any burden, and my mom does not know about my cancer. I don't want to hurt her feelings. That is why I come here alone. Well, I don't need anyone to help me come here because I can come here by myself." (Participant No.4).

"I don't have anything to need from my family. It is my life that I deal with, not the one who can live instead of me. I do not need anything. It would be annoying if someone is around me. I can walk by myself, so I don't need anyone to help me." (Participant No.5).

\section{Topic 4. Trust and Following kind medical team}

We categorized the trust issue about medical team into 3 codes; trust and follow, kind medical team, hope for high rate of full recovery with development of therapy method, etc.

Trust and follow: Participants think that medical team's speech and behavior are very important, and took attitude toward to entrust the treatment to the medical team. They showed their unlimited trust for the medical team claiming that they only follow the directions, because they do not have a medical knowledge. They said they follow the directions faithfully without questions.

"At first, I was actually scared of doing it. But it appears it is not. They did it for me, so all I should do is trust them. (Eyes with tears) Until now, I have trusted my doctor and nurse. If not, whom should I trust?" (Participant No.3).

"There is nothing to say about my treatment, because doctors are experts at this, and I don't know much about it. I follow the directions, and it is right to follow orders. I feel more relieved when I take the treatment at here compared to the one that I had at the other hospital. And I expect to have a better result." (Participant No.4).

"I don't have curious things about treatments or try to know. I just commit myself to them. They would do their best. What can I do, if I don't think in this way? I just eat, because I am told so. Doctors have their own purpose for this, right? I eat things that I should take." (Participant no.7).

Kind medical team: Participants were grateful about medical team's kindness, especially saying nice talk, and proud of National Cancer Center's medical team.

"Even if it is a government-established hospital, doctors and nurses are so kind and nice to me. I appreciate all the time." (Participant No.1).

"I appreciate even the nice word. I have never been in a bad or unwilling situation. They have treated me very nicely." (Participant No.3).

"It is so good to have this nice hospital. It is wonderful that this hospital saved so many people. People say we got a good hospital, and Korea is lucky to have it." (Participant No.2).
Hope for high rate of full recovery with development of therapy method: Participants expected to have a developed therapy method with more simple approaches thanks to doctors' positive research activities. They also want to be taken care of their cancers well.

"I think doctors should develop the method quickly. I want only this one. We should develop for our descendants. We should be cured with simple methods such as one shot of injection, pills and so on. As I experienced it, it is hard... If doctors take good care of this (lung cancer), there is nothing else I want more. What should I hope for unless I get cured?" (Participant No.5).

\section{Topic 5. Regret of past}

Participants was being regretful about their past. Especially, they regret smoking and putting aside their physical check-ups.

Regret of smoking: The participants who had been smoking seriously have decided to avoid second-hand smoking while recognizing the risk of smoking.

"I smoked a lot when I am driving... And I smoked a lot when I play game of go. I smoked too much. I smoked about two pack of cigarettes a day. Smoking was the main reason." (Participant No.4).

"I don't go near the smoking area. I regret a lot." (Participant No.1).

They show regrets about neglecting check-ups.

"My insurance cover the check-up once in two years. Nonghyup sent me here. I think something happened at that time. I was told to see a chief doctor, but I was busy to work, so I neglect it without any deep thought. If I were there, I would solve all the problems. But it has been 7 to 8 months since I have heard. I am guessing something would be changed, if I'd listened it." (Participant No.7).

\section{Topic 6. Wish for living long and healthy}

Participants had diverse expectations about their lives after recovery, and we categorized it into two codes. At first, they had a wish for living long while paying more specific attention like preparing special food in recuperation in the place of nice weather, when they finish the treatment, with regrets of smoking and neglecting the check-ups.

Want for nice weather, special food and exercise: Participants had a high interest in recuperation with nice weather, home remedy like fermented food, sweet wormwood and so on, after they finish the treatment. They put a specific effort such as exercise and meal to get over the cancer.

"I will go to somewhere with nice atmosphere, when my treatment is over. There are beach and national park. 
My family is researching this for now. They say I should it fermented food." (Participant No.2).

"I prepared many things like tonic for my body. I just don't use them because my doctor said not to do. I heard sweet wormwood is very good, so I planted three thousand clumps of it. I take care this, because I heard that it is good for the recovery and making enzymes later. I heard Sancai and something from the rocks that I can't remember the exact name, but it is good for body. Sancai is a new thing mentioned lately. It is coming from Myanmar and Himalaya. I bought them online after I saw them on the television last week. I only bought 50 clumps. It is not many. Also, I bought Mundungipul." (Participant No.9).

Wish for living long: Participant constantly wishes to live long without diseases. We saw his or her affection for healthy life hoping for no relapse or metastasis.

"I will carry this disease for my entire life, if I can't get full recovery. In fact, I want to stay with other people and sick (later) without further progress of cancer." (Participant No.1).

"I decided to put an effort not to get a relapse as I do my best on dietary therapy after the treatment, so I will deal with it well. Then, if I don't get full recovery, I just want not to get relapse. No relapse means full recovery to me. Shrinking would be better, but no longer growing is still fine. It would be good, if there were no metastasis. If so, there would be no impediment in life. Well, I should live until my little girl marries someone. My granddaughter is now 9 years old. I don't know I can live until she gets married. It is my hope." (Participant No.4).

\section{Discussion}

This work involved in-depth interviews and content analysis of the experiences of patients with 9 inoperable stage III NSCLC receiving CCRT. As of 2012, various drugs and treatments have been developed and used compared to 2013. The reason why we want to present this is that no matter what the treatment is, we believe that patients with inoperable stage III NSCLC who are sick will have the same wish for treatment. I think it would be very helpful for the medical staff to understand the phenomenon of what the patient's wish to be fully cured is expressed in what form and what action he/she takes.

This study comes up with 6 topics through analyzing qualitative content analysis after collecting the data from interviews to figure out related experiences of CCRT of 9 lung cancer patients. In the topic 1 , 'Fear of relapse with hoping for full recovery', there were 7 people out of 9 for 'hope for full recovery' as a participants' expectation in CCRT, and this result corresponds to the research about the nature of hope among Iranian cancer patients by Afrooz, et al. [12] that demon $94 \%$ of patients mentioned 'return to normal life' and 'complete healing of disease by drugs and physicians' as their main hope. 8 participants mostly answered 'they expect the full recovery', because their disease duration was short, as it had not exceeded 3 months since they started treatment with diagnosis. Therefore, for participants, it is very important to support their hope regardless of disease duration whether it is short or not. Also, participants had questions about treatment progress. At that time, no specific information about progression free survival or overall survival was provided in the agreement. CCRT proceeds for 5 days a week in 5 to 6 weeks, and interview with doctor takes once a week. In the end of the treatment, 1 month later, we observe curative effacement and side effects, as ambulatory care takes tri-monthly. We mainly observe the side effect during the treatment interview. Even though we cannot explain the effect during the treatment, we recognize the anxiety of participant and realize the need of various emotional supports to control it. Besides, the fear of relapse was also the emotion during the treatment, demonstrated by research of Kang, et al. [13] This means that patients are not only expecting the treatment but also seized with uncertainty, and they basically have a fear of relapse.

In the topic 2, 'Hardship of treatment despite pleasure', we can conclude it as it was the hope for full recovery as participants take the treatment everyday pleasantly regarding the treatment as enjoyment by thinking it helps them to recover. As CCRT gives patients the hope of full recovery, the continuity of treatment has an important meaning to participants. They pleasantly accepted the fact that they can get cared like the participants of this research, as the case of CCRT right after the diagnosis. As a symptom with regard to radiotherapy, the uncomfortable feelings of sore and burning for throat appeared in all 5 participants who had treated over than 12 times. It is an esophagitis symptom. It was reported there are esophagitis over than 3 degrees and radiation pneumonitis during CCRT [4]. In Pherbanbycq's study, [14] esophagitis was $28.1 \%$ and radiation pneumonitis was 7.9\%. In Kang, etc.'s study [4], esophagitis was $11.1 \%$ and radiation pneumonitis was $13.9 \%$. In this manner, esophagitis is a symptom that appears as CCRT continues, so, we can recognize the need of interest, explanation and proper mediation. Also, as radiotherapy continues, many patients expressed their inconvenience of fatigue and eating. According to Yang [10], fatigue is highest in 5 and 6 weeks of treatment and has largest increase in 2 weeks of treatment. Among patients of this study, the ones who complained fatigue were participants with 21 times of radiotherapy and 6 times of it. Participants said they had nausea, lethargy, dizziness, anemia and pain as related symptoms of chemotherapy, and it is hard and painful chemotherapy is repeated in 3 weeks. For cancer drugs, Etoposide and Cisplatin prescribed in tri-weekly basis, Cisplatin can give serious symptoms of nausea vomiting, as 
its daily dosage is larger than weekly dosage [4]. It is reported that patients with CCRT had neutropenia over than 3 degrees and anemia. As CCRT stipulates in the outpatient treatment, most participants located far away unless one who lived in 30 minutes away by car from hospital, so they should deal with the outpatient treatment in various ways. In other words, they used sanatorium, patient room , hospital ward, taxi, bus, driving. In Yang's study [10] of supportive nursing require from geriatric progressive lung cancer outpatients, participants' average age was 68 , and their highest need was help for visiting hospital. In this study, median age was 70 and average age was 68 . Even though participants required inpatient treatment appealing the difficulties of outpatient treatment, it is unnecessary in case of physical activity rage ECOG 1, and it is advisable to use diverse resources around hospital considering the balance of therapy and life. CCRT requires sufficient nutrition supply. The fatigue derived from radiotherapy and loss of appetite and vomiting from chemotherapy reduce weight of participants by debasing stamina. Besides, in lung cancer case, there are many cases that experiencing loss of weight, and it is possible to bear the treatment to the end of it when there is enough nutrition supply retaining the weight. Even if participants are educated not to eat hot, boiling and stimulating food when they are with CCRT, they continuously have curiosity about which food they should eat in fact, especially, they are interested in home remedies good for cancer. Thus, medical team should continuously pay attention to participants' difficulties of swallowing and throat pain during the treatment and evaluate the symptoms and guide them to use developed and commercialized high-protein nutrition drinks actively.

In topic 3, 'Sense of guilt about sufficient help and sense of independence with self-management', participants were having treatment while thinking that they must live thanks to their family's sufficient supports. Considering Afrooz, et al. [12] reported that the source of hope in cancer patients was spiritual resources, family/health care worker's support. Moreover, they had a sense of guilt despite of sufficient family supports. Some participants had a treatment without letting family know about the therapy, and some had an outpatient treatment alone as they can control themselves without any family's help. We think, however, CCRT needs family support as they get fatigue and exhausted as time goes by.

In the topic 4, 'Trust and Following kind medical team', participants had a full faith that makes them to follow doctors' orders by letting themselves to doctors. Afrooz, et al. [12] study, cancer patients thought of health care worker as a source of hope, which could mean that the patient trusts the doctor, which would increase their compliance with treatment. This means supports from medical teams affect quality of life. Moreover, participants were touched by kind words and behavior of doctors, nurses, radiological technologists, and this kindness is the important fact for patients and family to recognize medical team as good
[15]. They follow doctors, as they trusted, meanwhile, they expected new technology for the cure through active studies of doctors.

In the topic 5, "Regret of past", participants regret their smoking in the past as the smoking is known as the cause of lung cancer. Lee and Kim's study [16] shows patients considered smoking was the cause and had a stigma. As we have observed participants' regrets of discovering cancer too late because of neglecting the instruction to see doctors after their check-up, the public relations and education about cancer prevention and early detection are necessary.

In the topic 6, "Wish for living long and healthy", participants were considering preparation for recuperation in nice weather, preparation for fermented food and diverse home remedies, exercising and meals and so on. This behavior corresponds with Lim, et al. [17] research that shows behaviors of breast and colorectal cancer survivors had the effort and willingness to change food and eating habits, find suitable exercise, and use folk remedies to manage their health. In specific, participants wanted not to sick when they get CCRT without thinking the 5 years of survival rate of lung cancer which is short, and showed their earnest hope to live over than 5 or 10 years without degeneration until their granddaughter or daughter get married. Medical teams regard this earnest hope for life of patients importantly. It means there should be a need for active support to let them have best treatment with their family.

\section{Conclusion and suggestion}

To figure out the related experiences of CCRT with 9 lung cancer patients, this study collected the data through deep interviews and analyzed by using qualitative content analysis and tried to utilize it in nursing intervention.

The experiences of participants were demonstrated in 6 topics which are "Fear of relapse with hoping for full recovery", "Hardship of treatment despite pleasure", "Sense of guilt about sufficient help and sense of independence with self-management", "Trust and Following kind medical team", "Regret of past", "Wish for living long and healthy". Considering the result of research, lung cancer patients with CCRT are taking therapies pleasantly with a hope for full recovery when they are treated, and have anxiety about relapse at the same time, so, there should be diverse emotional supports to control this. They also have various physical difficulties because of radiotherapy and chemotherapy; thus, there should be a physical symptom control and a support in accordance with a treatment and time. In particular, considering characteristics of patients who are from nationwide, the nursing intervention interested in places for outpatient treatment and guiding in advance is necessary. Lastly, considering that patients are having affections about their lives after the treatment and putting various efforts, we need an emotional support and a supply of proper nursing information. 
Based on the result of study, I would like to give suggestions as follow.

First, we need to provide the nursing intervention by making requests from patients with CCRT into a nursing education document and evaluate the effects.

Second, we need to study precisely what are the symptoms experienced by patients, the emotional difficulties and the practical help for patients having outpatient treatment continuously after the end of CCRT.

\section{References}

1. National Cancer Information Center (NCIC). Cancer Incidence Rate. 2021. https://www.cancer.go.kr/lay1/S1T648C650/contents.do

2. National Cancer Information Center (NCIC). Cancer Survival Rate. 2019. https://www.cancer. go.kr/lay1/program/S1T211C215/cancer / view/do?cancer_seg $=5237 \&$ menu_seg $=5253$

3. National Comprehensive Cancer Network. NCCN guidelines version 3, 2014. Non-small cell lung cancer. Fort Wahington, PA: National Comprehensive Cancer Networ. 2014.

http://www.nccn.org/professionals/physician_gls/pdf/nscl.pdf

4. Kang KM, Jeong BK, Ha IB, Chai GY, Lee GW, et al. Concurrent chemoradiotherapy for elderly patients with stage III non-small cell lung cancer. Radiat oncol J. 2012; 30: 140-145.

PubMed: https://pubmed.ncbi.nlm.nih.gov/23170293/

5. Noh JM, Ahn YC, Lee HB, Pyo HR, Kim BK, et al. Definitive biomodality concurrent chemoradiotherapy in patients with inoperable N2-positive stage IIIA non-small cell lung cancer. Cancer Res Treatment. 2015; 47: $645-652$

6. Lee YJ, Han JY, Moon SH, Nam BH, Lim KY, et al. Incorporating Erlotinib or Irinotecan Plus Cisplatin into chemoradiotherapy for Stage III Non-small cell lung cancer according to EGFR mutation status. Cancer Res Treatment. 2017; 49: 981-989.

PubMed: https://pubmed.ncbi.nlm.nih.gov/28111430/

7. Cheema PK, Rothenstein J, Melosky B, Brade A, Hirsh V. Perspectives on treatment advanced for stage III locally advanced unresectable non-small-cell lung cancer. Current Oncol. 2019; 26: 37-42. PubMed: https://pubmed.ncbi.nlm.nih.gov/30853796/

8. Hogle WP. The state of the art in radiation therapy. Seminars in Oncology Nursing. 2006; 22: 212-220.

9. Hickok JT, Morrow GR, Roscoe JA, Mustian K, Okunieff O. Occurrence, severity, and longitudinal course of twelve common symptoms in 1129 consecutive patients during radiotherapy for cancer. J Pain Symptom Management. 2005; 30: 433-442.

PubMed: https://pubmed.ncbi.nlm.nih.gov/16310617/

10. Yang JC, Chung WK. Psychology and quality of life in cancer patients on radiation therapy. J Korean Soc Ther Radiol Oncol. 2004; 22: 271-279.

11. Sandelowski $M$. The problem of rigor in qualitative research. Adv Nurs Sci. 1986; 8: 27-37.

PubMed: https://pubmed.ncbi.nlm.nih.gov/3083765/

12. Afrooz R, Rahmani A, Zamanzadeh V, Abdulahzadeh F, Azadi A, et al. The nature hope among Iranian cancer patients. Asia Pacific J Cancer Prevent. 2014; 15: 9307-9312.

13. Kang DB, Cho JH, Kim IR, Kim MK, Kim WS, et al. Health-related quality of life in Non-Hodgkin Lymphoma survivor: A prospective cohort study. Cancer Res Treatment. 2018; 50: 1051-1063. PubMed: https://pubmed.ncbi.nlm.nih.gov/29121713/

14. Pherbanbycq E, Spoelstra FOB, Verbakel W, Postmus P, Melissant $\mathrm{C}$, et al. Outcomes of concurrent chemoradiotherapy in patients with stage III non-small-cell lung cancer and significant comorbidity. Ann Oncol. 2011; 22: 132-138.

PubMed: https://pubmed.ncbi.nlm.nih.gov/20595452/

15. Cho NY, Han SS, Hong YS, Um YR. Attributes perceived by cancer patients as a good nurse. Clin Nurs Res. 2006; 11: 149-162.

16. Lee JL. Kim KS. The relationships between stigma, distress, and quality of life in patients with lung cancer. Asian Oncol Nurs. 2011; 15: 237-246.

17. Lim JW, Yang EJ, Sohn SR, Lee JS, Sohn JH. A qualitative study on perceptions and practical experiences regarding survivorship care for breast and colorectal cancer survivors and families. Health Soc Welfare Rev. 2016; 36: 511-543. 